\title{
REVISTAMATËRIA
}

\section{Avaliação da taxa de corrosão do aço carbono 1040 em solo por modulação de frequência eletroquímica (EFM)}

\author{
Evaluation of corrosion rate of 1040 carbon \\ steel in soil by electrochemical frequency \\ modulation (EFM)
}

\author{
Tiago Scheffer de Matos ${ }^{1,2}$, Kleber Franke Portella ${ }^{1,2}$ \\ Sérgio Luiz Henke ${ }^{3}$, Mariana D’Orey Gaivão Portella Bragança ${ }^{1}$ \\ Alessandro Cesar de Sousa Berrêdo ${ }^{4}$
}

\footnotetext{
${ }^{1}$ Lactec - Rodovia BR-116, km 98, 8.813, CEP: 81531-980, Curitiba, PR, Brasil.

${ }^{2}$ Universidade Federal do Paraná, Programa de Pós Graduação em Engenharia e Ciências dos Materiais-PIPE - Av. Cel. Francisco H. dos Santos, 100, CEP: 81530-000, Curitiba, PR, Brasil.

${ }^{3}$ Universidade Federal do Paraná, Departamento de Engenharia Mecânica - Av. Cel. Francisco H. dos Santos, 100, CEP: 81530-000, Curitiba, PR, Brasil.

${ }^{4}$ Transmissora Aliança de Energia Elétrica S.A. (TAESA) - Praça Quinze de novembro, 20, CEP: 20010-010, Rio de Janeiro, RJ, Brasil.

e-mail: tsmatosqui@yahoo.com.br, portella@lactec.org.br, mariana.portella@lactec.org.br, henke@ufpr.br, alessandro.berredo@taesa.com.br
}

\section{RESUMO}

A taxa de corrosão instantânea do aço carbono 1040 enterrado em solo, por 6 meses, foi medida por meio da técnica de modulação de frequência eletroquímica (EFM) e pela resistência à polarização linear (LPR). Os resultados foram comparados entre si e com os dados de taxa de corrosão obtidos pela análise de conjuntos de materiais similares e sob mesmo tempo de exposição em campo. Os valores obtidos por EFM foram de $(0,088 \pm 0,008) \mathrm{mm} / \mathrm{ano}$ e, por LPR, de $(0,075 \pm 0,005) \mathrm{mm} / \mathrm{ano}$. Já, pela perda de massa, foram calculadas variações de espessura deste material, de $(0,064 \pm 0,016) \mathrm{mm} / \mathrm{ano}$. Assim, nas condições experimentais impostas pode-se inferir que os resultados obtidos pela técnica EFM, serviram para diagnosticar o desempenho do aço carbono 1040 submetido a um processo de corrosão em solo.

Palavras-chave: Taxa de corrosão, aço carbono, solo, EFM, LPR.

\section{ABSTRACT}

The instantaneous corrosion rate of 1040 carbon steel buried in soil was measured by electrochemical frequency modulation (EFM) and linear polarization resistance (LPR). The results were compared to each other and to the corrosion rate data obtained by the analysis of corrosion coupons exposed in field. The values of instantaneous corrosion rate found in the soil by EFM were $0.088 \pm 0.008 \mathrm{~mm} /$ year, by LPR of $0.075 \pm$ $0.005 \mathrm{~mm} /$ year. The corrosion rate values obtained by weight loss of corrosion coupons were $0.064 \pm 0.016$ $\mathrm{mm} /$ year. Thus, in the experimental conditions imposed it can be inferred that the results obtained by the EFM technique served to diagnose the performance of 1040 carbon steel subjected to a soil corrosion process. Keywords: Corrosion rate, carbon steel, soil, EFM, LPR.

\section{INTRODUÇÃO}

A degradação ou a corrosão dos metais enterrados em solo tem trazido preocupação em relação a aspectos como a segurança e os custos em manutenção em oleodutos, minerodutos, tanques de estocagem de combustível e em sistemas de ancoragem de torres de linhas de transmissão [1-4]. Isto é, em muitos dos casos, con- 
sequente da complexidade do processo envolvido, devido ao grande número de variáveis, como a umidade, a temperatura, o teor de sais dissolvidos, a resistividade, o pH, entre outros, como a própria natureza dos produtos formados em sua superfície, já que estes são resultantes do meio e da sua composição química original [5-10]. Como relevância, a camada superficial formada pode até mesmo ser benéfica, reduzindo ou atenuando a progressão da corrosão por um processo protetivo, melhorando a sua estabilidade neste ambiente [11-14].

$\mathrm{Na}$ investigação dos mecanismos de corrosão metálica em ambientes variados vem sendo utilizadas diversas técnicas eletroquímicas, entretanto a grande maioria destas necessita do conhecimento prévio das constantes anódica e catódica de Tafel ( $\beta$ a e $\beta c$ ) [15]. Assim, tem-se como exemplo, a técnica de resistência a polarização linear (LPR) que vem sendo utilizada para avaliação de materiais metálicos em diversos estudos principalmente por ser considerada uma técnica não destrutiva, onde os valores de sobrepotenciais aplicados $(10 \mathrm{a} 15 \mathrm{mV})$ não alteram a superfície do material. Entretanto, nas medidas quantitativas de taxa de corrosão é necessário o conhecimento dos valores de $\beta \alpha$ e $\beta c$ [2], [15], [16].

A técnica de modulação de frequência eletroquímica tem ganho destaque como uma ferramenta promissora para a avaliação da corrosão, pois a taxa de corrosão e as constantes de Tafel podem ser calculadas pela análise dos sinais harmônicos e intermodulares gerados pelo processo de corrosão, quando este é perturbado por sinal senoidal superposto de baixa amplitude (10 a $25 \mathrm{mV}$ ) [17-19]. Neste caso, os sinais harmônicos e intermodulares podem trazer outras informações além da taxa de corrosão e dos coeficientes de Tafel, como por exemplo, nos trabalhos desenvolvidos por Rauf e Bogaerts [20], [21], onde a relação entre esses sinais foi utilizada para detectar e diferenciar a corrosão generalizada, em frestas e por pites.

Nesta investigação, foi estudado o desempenho ou a eficácia da técnica de modulação de frequência eletroquímica (EFM) na análise da taxa de corrosão de corpos de prova cilíndricos de aço carbono 1040 enterrados em solo, por cerca de 6 meses, confinados em células eletroquímicas de medida, mas, mantidas em ambiente natural externo, sendo seus resultados confrontados com os das medidas realizadas por LPR, nos mesmos sistemas. Como proposta adicional, também foi efetuada a análise de corpos de prova similares pela perda de massa média resultante, dos mesmos, quando expostos ou enterrados no mesmo tipo de solo e período de tempo, porém, sem seu confinamento experimental, de modo a se poder inferir sobre a influência do meio na resposta do conjunto de análise.

\section{MATERIAIS E MÉTODOS}

\subsection{Materiais}

Hastes de aço carbono 1040, em formato cilíndrico, com $8 \mathrm{~cm}$ de comprimento e $1 \mathrm{~cm}$ de diâmetro, sem tratamento superficial, preparadas conforme recomendação da norma ASTM G-01 (2017) [22], foram utilizadas nos ensaios eletroquímicos de corrosão em solo. A composição química elementar do metal, pode ser visualizada na Tabela 1.

Tabela 1: Composição química do aço carbono 1040, utilizado nos ensaios de corrosão em solo.

\begin{tabular}{c|c|c|c|c|c|c}
\hline \multicolumn{7}{c}{ ELEMENTOS (\% MASSA) } \\
\hline & $\mathrm{C}$ & $\mathrm{Si}$ & $\mathrm{Mn}$ & $\mathrm{P}$ & $\mathrm{S}$ & $\mathrm{Fe}$ \\
\hline HASTE ANALISADA & 0,47 & 0,25 & 0,66 & 0,022 & 0,012 & BALANÇO \\
\hline
\end{tabular}

O solo utilizado nas análises eletroquímicas foi coletado em uma região próxima a uma torre estaiada de uma linha de transmissão de energia elétrica (LT) no interior do nordeste do Brasil, em estudo de caso. Este solo continha um teor de cloreto e sulfato menor que $5 \mathrm{mg} / \mathrm{kg}$. Nesse mesmo local, foram instaladas hastes de aço carbono similares e de mesmo material para as análises da taxa de corrosão pela técnica de perda de massa.

As extremidades do cilindro de aço carbono foram isoladas do contato com o ambiente com fita auto fusão, de modo que uma área média de $(8,0 \pm 0,2) \mathrm{cm}^{2}$ na região central do cilindro ficou exposta para todos os sistemas em estudo. Este, foi inserido pela tampa de um porta-amostra de $350 \mathrm{~mL}$ com as marcações para cada eletrodo, conforme modelo ilustrativo mostrado na Figura 1.

\subsection{Ensaios de eletroquímicos}

As análises de EFM e LPR foram realizadas, em duplicata, durante 3 dias consecutivos, na célula eletroquímica montada com três eletrodos em potencial de circuito aberto (OCP), em solo saturado com água, após 6 
meses de exposição natural na cidade de Curitiba- PR. Os ensaios de EFM foram conduzidos pela aplicação de duas ondas senoidais superpostas de frequência $\omega_{1}$ e $\omega_{2}$ de 2 e $5 \mathrm{~Hz}$ respectivamente, com uma amplitude de sinal de $25 \mathrm{mV}$ (rms). As análises de LPR foram realizadas com a utilização de sobreprotenciais anódico e catódico de $15 \mathrm{mV}$ com uma taxa de varredura de $0,2 \mathrm{mV} / \mathrm{s}$. No cálculo da taxa de corrosão foram utilizados os valores de $\beta$ a e $\beta$ c obtidos por EFM. Para as medidas, foi utilizado um potenciostato/galvanostato modelo Reference 600+ da marca GAMRY para a aquisição dos dados em laboratório, com temperatura ambiente controlada em $(23 \pm 2)^{\circ} \mathrm{C}$.
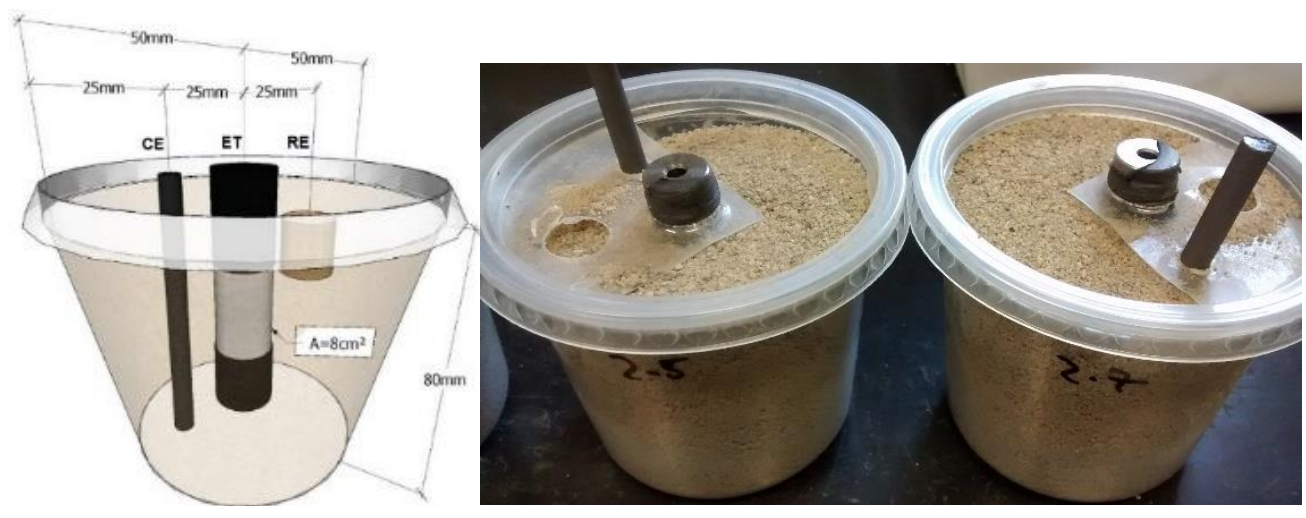

Figura 1: Desenho ilustrativo do sistema de medida (esquerda) e imagem da célula eletroquímica utilizada (direita) para as medidas de taxa de corrosão por EFM e LPR, em amostras cilíndricas de aço carbono 1040, encapsuladas, em solo.

\subsection{Ensaios de perda de massa}

Os cupons de corrosão foram preparados, identificados e analisados conforme a norma ASTM G-01 (2017) [22]. Os mesmos foram enterrados a cerca de $2 \mathrm{~m}$ da base do sistema de ancoragem da LT estaiada, localizada no interior do nordeste do Brasil, sendo 4 cupons, distantes $50 \mathrm{~cm}$ de uma estaca de identificação. Parte estatística destes cupons foi coletada e analisada, após 6 meses de sua exposição em campo.

\section{RESULTADOS E DISCUSSÃO}

As análises de EFM e LPR foram executadas nas amostras de aço carbono enterradas no solo, estando expostas em ambiente natural externo por 6 meses. Na Figura 2 é possível visualizar o gráfico obtido na análise de LPR, onde a porção linear foi utilizada para o cálculo da resistência à polarização e, consequentemente, da taxa de corrosão. Os valores dos coeficientes de correlação de Pearson e R-quadrado obtidos pelo ajuste linear dos dados experimentais de LPR indicaram haver um grau de correlação adequado entre os dados.

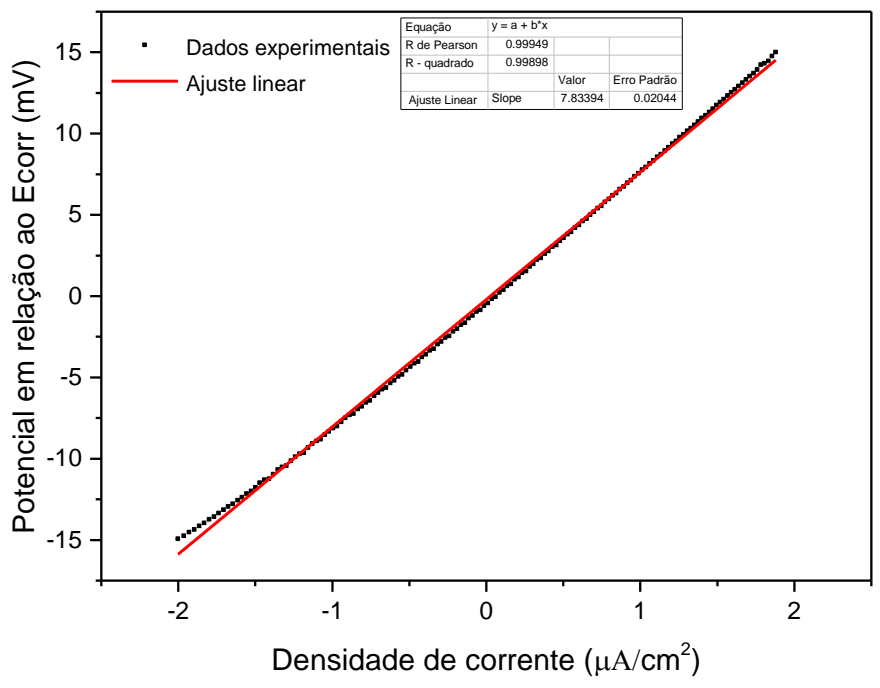

Figura 2: Resistência a polarização do aço carbono enterrado em solo após 6 meses de exposição em ambiente natural. 
Na Figura 3 é possível visualizar os valores de densidade de corrente no domínio da frequência, gerados pela perturbação do sistema $\left(\omega_{1}=2 \mathrm{~Hz}\right.$ e $\left.\omega_{2}=5 \mathrm{~Hz}\right)$, além dos sinais harmônicos e intermodulares. Se a corrosão fosse um processo linear, apenas os sinais utilizados para perturbar o sistema seriam obtidos no espectro de corrente no domínio da frequência. Como isso não acontece, a resposta ao sinal aplicado é obtida em um número maior de frequências além daquela aplicada ao sistema [17]. Observaram-se, valores de densidade de corrente maiores nas frequências de perturbação $\omega_{1}$ e $\omega_{2}$, além dos sinais intermodulares $\left(2 \omega_{1}+\omega_{2}\right)$ e $\left(2 \omega_{2}+\omega 1\right)$. Já, os sinais harmônicos $2 \omega_{1}$ e $2 \omega_{2}$ tiveram menores valores de densidade de corrente, o que corroborou com os dados obtidos em alguns trabalhos que indicaram a correlação entre a magnitude dos sinais de corrente em frequências intermodulares e dos sinais harmônicos [17], [21].

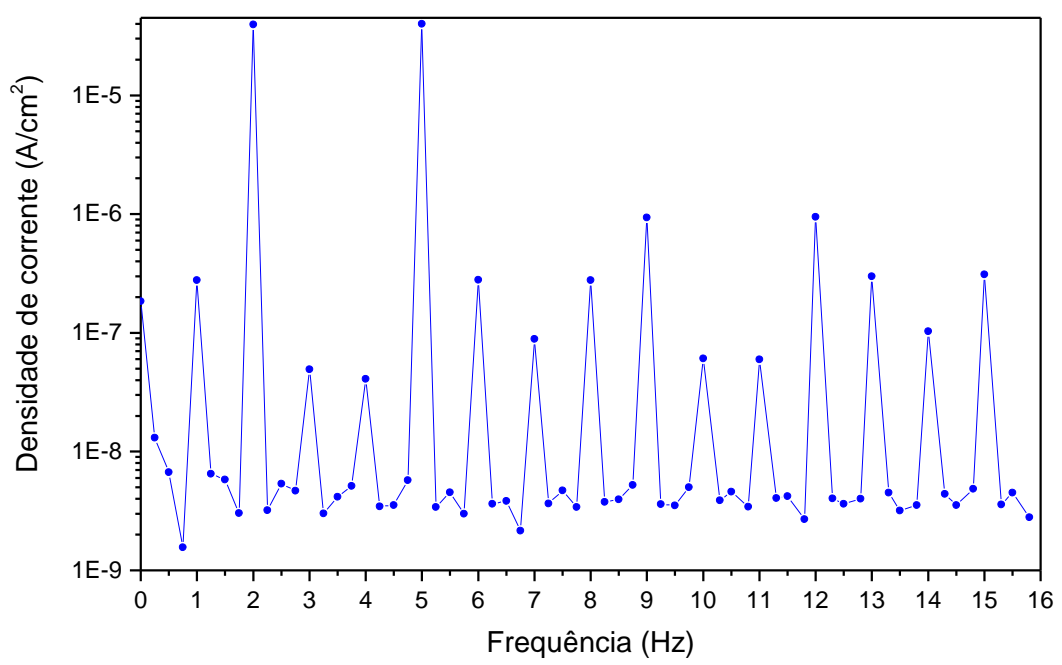

Figura 3: Espectro de frequência da resposta EFM de corrente do aço carbono em solo após 6 meses de exposição em ambiente natural.

Os resultados de taxa de corrosão obtidos por EFM e LPR podem ser visualizados na Figura 4. A taxa de corrosão por LPR teve resultados menores nas análises realizadas nos 3 dias consecutivos, entretanto, essa diferença ficou na ordem de $0,01 \mathrm{~mm} /$ ano. Ambas as técnicas tiveram pequena dispersão dos resultados, o que mostrando a precisão das técnicas eletroquímicas para realizar as medidas de taxa de corrosão. Além disso, o aumento no valor da taxa de corrosão instantânea no segundo dia de análise, em ambas as técnicas, pode ser devido a adição de água para a saturação do solo realizada no primeiro dia.

Foi possível observar que no primeiro e terceiro dia de análises os resultados estiveram muito próximos, o que indica a estabilização na taxa de corrosão após a adição de água para saturação do solo.

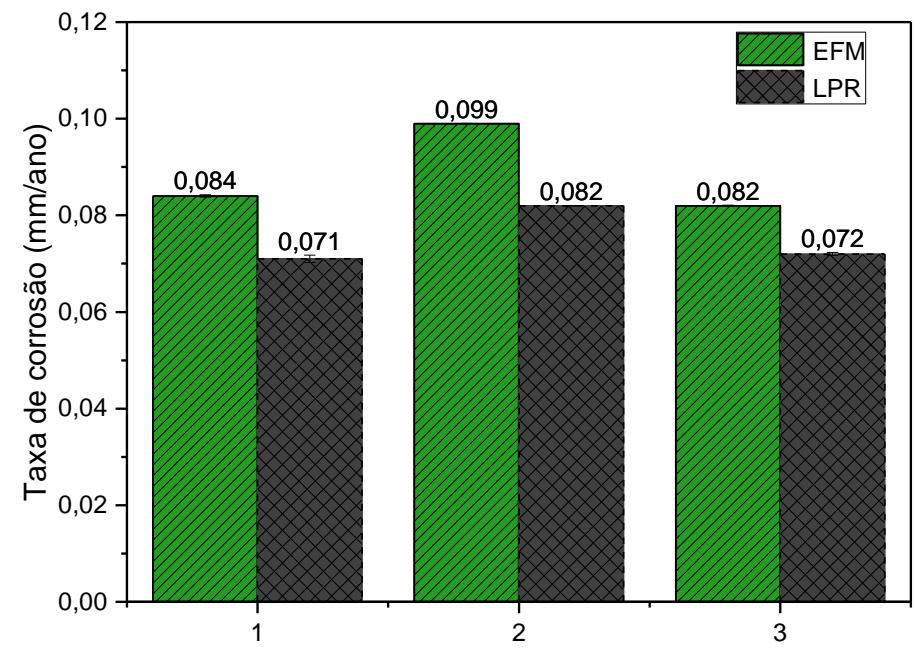

Figura 4: Taxa de corrosão do aço carbono em solo obtidas por EFM e LPR em três dias consecutivos. 
Os valores médios de taxa de corrosão instantânea encontrados nos 3 dias consecutivos de análises pelas técnicas de EFM e de LPR, além dos resultados de taxa de corrosão obtidos pela tradicional técnica de perda de massa podem ser visualizados na Figura 5. Os resultados encontrados indicaram uma boa correlação, não havendo diferenças significativas entre as técnicas utilizadas segundo a análise de variância. Embora os resultados de perda de massa tenham tido uma dispersão elevada, quando comparados com as técnicas eletroquímicas, os valores de taxa de corrosão estiveram na mesma ordem de grandeza. A análise de perda de massa por meio dos cupons de corrosão foi realizada em um ambiente com condições climáticas diferentes, e apesar disso, os resultados ficaram próximos a aqueles encontrados pelas técnicas eletroquímicas. Esses dados além de indicarem a correlação entre as técnicas utilizadas em laboratório, pode-se, por seu intermédio, avaliar a influência das características do solo no processo de corrosão do aço carbono, pois mesmo em climas e condições diferentes, as taxas de corrosão ficaram próximas, durante o período de estudo. Na Figura 6 é possível visualizar a média pluviométrica dos locais onde foram realizados os ensaios de exposição do aço carbono em laboratório e campo.

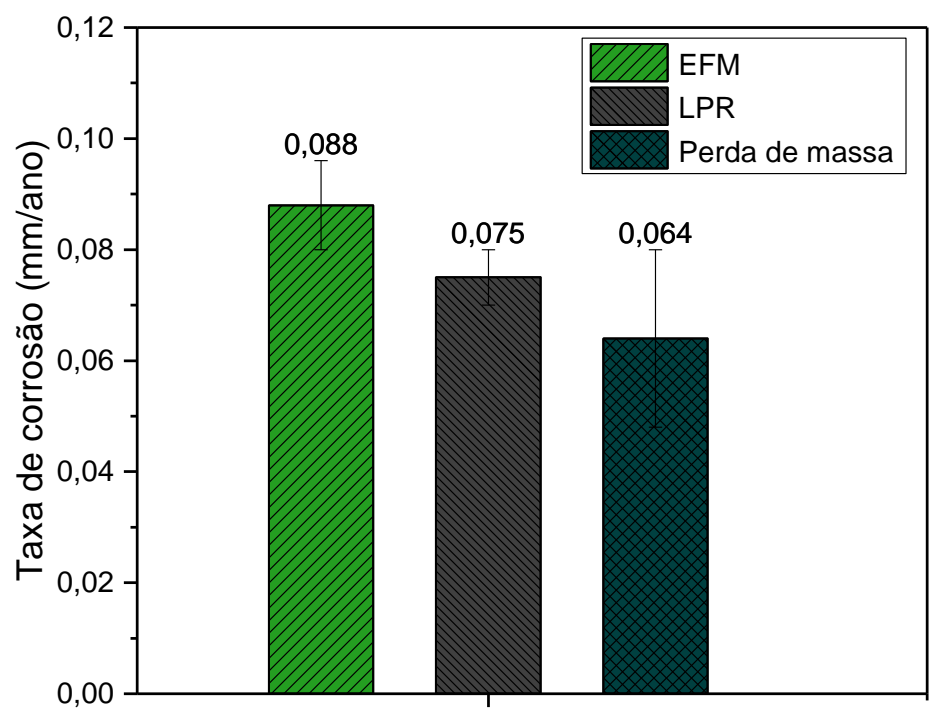

Figura 5: Resultado dos valores de taxa de corrosão obtidos por EFM, LPR e perda de massa em amostras de aço carbono em solo.

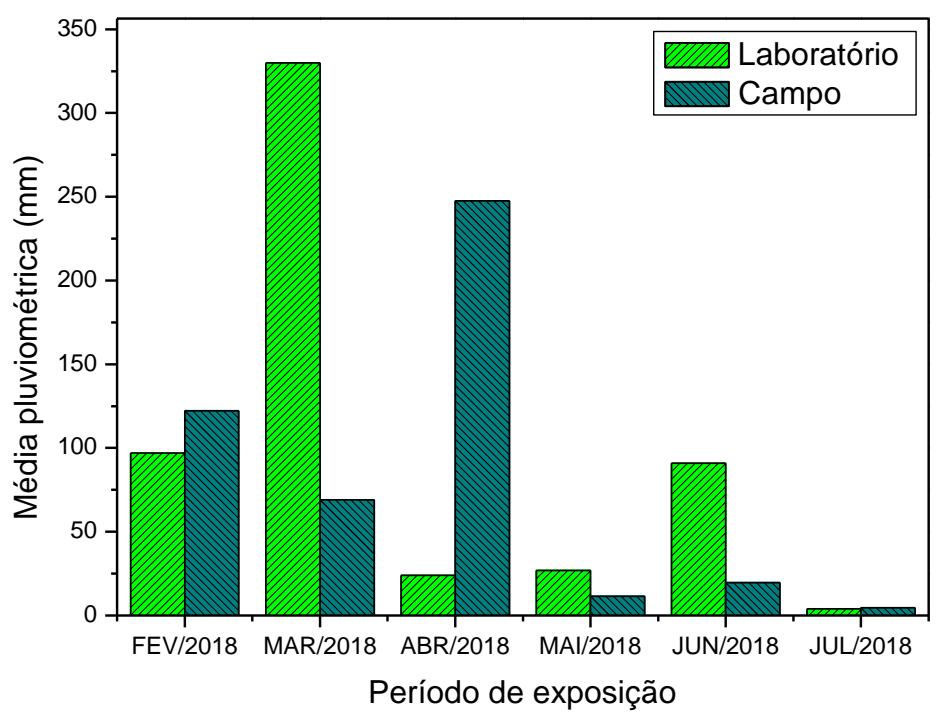

Figura 6: Média pluviométrica dos locais onde foram realizados os ensaios eletroquímicos (laboratório) e exposição dos cupons de corrosão (campo), no período de fevereiro a julho de 2018. 
Os trabalhos realizados até o momento indicaram boas correlações entre a técnica de EFM e as demais técnicas eletroquímicas em diversos meios aquosos [17], entretanto para alguns casos ocorreram divergências nos valores de taxa de corrosão [19]. No caso da avaliação da taxa de corrosão do aço carbono 1040 em solo, os valores, nessa faixa de taxa de corrosão, ficaram na mesma ordem de grandeza entre as técnicas de EFM e LPR.

\section{CONCLUSÕES}

Os valores obtidos para a taxa de corrosão do aço carbono 1040 em solo por EFM foram de $(0,088 \pm 0,008)$ $\mathrm{mm} /$ ano e, por LPR, de $(0,075 \pm 0,005) \mathrm{mm} / \mathrm{ano}$. Já, pela perda de massa, foram calculadas variações de espessura deste material, de $(0,064 \pm 0,016) \mathrm{mm} /$ ano. Assim, nas condições experimentais impostas pode-se inferir que os resultados obtidos pela técnica EFM, serviram para diagnosticar o desempenho deste material a um processo de corrosão em solo.

O resultado deste estudo evidenciou a precisão das técnicas eletroquímicas de EFM e LPR em relação a tradicional técnica de perda de massa. Além disso, os resultados indicaram que a técnica de EFM pode ser utilizada para o monitoramento da taxa de corrosão do aço carbono em solo com a vantagem de não ser necessária a realização de análises adicionais para a obtenção das constantes de Tafel.

\section{AGRADECIMENTOS}

Os autores agradecem à infraestrutura e ao apoio em recursos humanos e financiamento à TAESA, projeto P\&D 5012-0045/2016, à ANEEL, aos Institutos LACTEC, ao CNPq Lei 8010/90 (LI 15/2187214-1; LI 14/4695814-5; LI14/3410726-9), ao P\&D COPEL 6491-0301/2013, ao CNPq/PIBIT e de bolsa DT, processo 302672/2016-8, e à Universidade Federal do Paraná - PIPE/UFPR.

\section{BIBLIOGRAFIA}

[1] SANTOS, M.C., et al., "Investigação do processo de corrosão causado pela polpa de bauxita em mineroduto de aço carbono", Matéria (Rio J.), v. 20, n. 1, pp. 178-184, 2015.

[2] WANG, S. et al., "Field corrosion characterization of soil corrosion of X70 pipeline steel in a red clay soil,” Progress in Natural Science: Materials International, v. 25, n. 3, pp. 242-250, 2015.

[3] BERENGUER, R.A., et al., "Guy Structure with Galvanic Corrosion: Case Study,” Journal of Civil Engineering and Architecture, v. 10, n. 7, pp. 792-797, 2016.

[4] RIEDER, E.S., et al., "Investigação dos principais processos de corrosão em estações de energia elétrica do Estado do RS,"., Matéria (Rio J.), v. 14, n. 3, pp. 1000-1014, 2009.

[5] OGUZIE, E.E., et al., "Monitoring the corrosion susceptibility of mild steel in varied soil textures by corrosion product count technique," Materials Chemistry and Physics, vol. 84, n. 1, pp. 1-6, 2004.

[6] SORIANO, C., ALFANTAZI, A., "Corrosion behavior of galvanized steel due to typical soil organics", Construction and Building Materials”, v. 102, pp. 904-912, 2016.

[7] STOTT, J.F.D., et al., "Corrosion in Soils", In: abdullahi A. A., Reference Module in Materials Science and Materials Engineering, New York, Elsevier, pp. 1-20, 2018.

[8] NISHIMURA, T. "Rust Formation Mechanism on Low Alloy Steels after Exposure Test in High $\mathrm{Cl}^{-}$and High SOx Environment”, Materials (Basel)., v. 10, n. 2, p. 199, 2017.

[9] REFAIT, P.H., et al., "Corrosion and cathodic protection of carbon steel in the tidal zone: Products, mechanisms and kinetics”, Corrosion Science., v. 90, pp. 375-382, 2015.

[10] YAN, M., et al., "Role of Fe oxides in corrosion of pipeline steel in a red clay soil," Corrosion Science, v. 80, pp. 309-317, 2014.

[11] KUMAR, A.V.R., BALASUBRAMANIAM, R., "Corrosion product analysis of corrosion resistant ancient indian iron", Corrosion Science, v. 40, n. 7, pp. 1169-1178, 1998.

[12] ALCÁNTARA, J., et al., "Marine atmospheric corrosion of carbon steel: A review," Materials (Basel)., v. 10, n. 4, 2017.

[13] MA, Y., et al., "The effect of $\beta-\mathrm{FeOOH}$ on the corrosion behavior of low carbon steel exposed in tropic marine environment", Materials Chemistry and Physics, v. 112, n. 3, pp. 844-852, 2008. 
[14] ALCÁNTARA, J., et al., "An attempt to classify the morphologies presented by different rust phases formed during the exposure of carbon steel to marine atmospheres", Materials Characterization, v. 118, pp. 65-78, 2016.

[15] POPOV, B.N., CORROSION ENGINEERING Principles and Solved Problems, New York, Elsevier, 2015.

[16] ELSENER, B., et al., "A non-destructive in-situ approach to monitor corrosion inside historical brass wind instruments”, Microchemical Journal, v. 124, pp. 757-764, 2016.

[17] BOSCH, R. W., et al., "Electrochemical frequency modulation: a new electrochemical technique for online corrosion monitoring”, Corrosion, v. 57, n. 1, pp. 60-70, 2001.

[18] KUŞ E. AND MANSFELD, F., "An evaluation of the electrochemical frequency modulation (EFM) technique”, Corrosion Science, v. 48, n. 4, pp. 965-979, 2006.

[19] OBOT I. B., ONYEACHU, I. B., "Electrochemical frequency modulation (EFM) technique: Theory and recent practical applications in corrosion research", Journal of Molecular Liquids, v. 249, pp. 83-96, 2018.

[20] RAUF, A., BOGAERTS, W.F., "Monitoring of crevice corrosion with the electrochemical frequency modulation technique," Electrochimica Acta, v. 54, n. 28, pp. 7357-7363, 2009.

[21] RAUF, A., BOGAERTS, W.F., "Employing electrochemical frequency modulation for pitting corrosion”, Corrosion Science, v. 52, n. 9, pp. 2773-2785, 2010.

[22] ASTM G 01, "Standard Practice for Preparing, Cleaning, and Evaluating Corrosion Test", American. Society for Testing and Materials, v. 90, pp. 1-9, 2017.

\section{ORCID}

Tiago Scheffer de Matos

Kleber Franke Portella

Sérgio Luiz Henke

Mariana D’Orey Gaivão Portella Bragança

Alessandro Cesar de Sousa Berrêdo https://orcid.org/0000-0002-6383-1835

http://orcid.org/0000-0002-0631-9990

http://orcid.org/0000-0002-4322-0930

https://orcid.org/0000-0002-2702-7196

https://orcid.org/0000-0002-4541-7809 